AperTO - Archivio Istituzionale Open Access dell'Università di Torino

\title{
Laparoscopic Nephron-Sparing Calycectomy for Treating Fraley's Syndrome
}

\section{This is the author's manuscript}

Original Citation:

\section{Availability:}

This version is available http://hdl.handle.net/2318/1670403

since 2018-07-05T17:38:43Z

Published version:

DOI:10.1159/000486628

Terms of use:

Open Access

Anyone can freely access the full text of works made available as "Open Access". Works made available under a Creative Commons license can be used according to the terms and conditions of said license. Use of all other works requires consent of the right holder (author or publisher) if not exempted from copyright protection by the applicable law. 
This is the author's final version of the contribution published as:

Laparoscopic Nephron-Sparing Calycectomy for Treating Fraley's Syndrome

Francesco Porpiglia, Riccardo Bertolo, Enrico Checcucci, Daniele Amparore, Matteo Manfredi, Cristian Fiori

Urol Int. 2018;100(2):134-138.

doi: $10.1159 / 000486628$.

The publisher's version is available at:

[https://www.karger.com/Article/Abstract/486628]

When citing, please refer to the published version.

Link to this full text:

[http://hdl.handle.net/ hdl:2318/1670403] 


\title{
Laparoscopic Nephron-Sparing Calycectomy for Treating Fraley's Syndrome
}

\author{
Francesco Porpiglia Riccardo Bertolo Enrico Checcucci Daniele Amparore \\ Matteo Manfredi Cristian Fiori \\ Division of Urology, Department of Oncology, School of Medicine, San Luigi Gonzaga Hospital, University of Turin, \\ Turin, Italy

\section{Keywords} \\ Laparoscopy $\cdot$ Retroperitoneoscopy $\cdot$ Kidney $\cdot$ Nephron sparing \\ surgery $\cdot$ Renal pelvis · Stenosis · Surgical repair · Surgical technique
}

\begin{abstract}
Background/Aims/Objectives: Various nephron-sparing approaches were described as part of surgical management for Fraley's syndrome, a rare anatomic variant of the renal vascular anatomy that compresses the upper pole infundibulum resulting in an upper calyceal obstruction and dilatation, with symptoms of flank pain and hematuria. To date, descriptions of minimally invasive correction techniques are anecdotal. Methods: A retroperitoneal pure laparoscopic approach using the nephron-sparing technique was chosen in the presented case. Results: In this report, we demonstrated that if laparoscopic calycectomy is performed without clamping of renal branches, parenchymal ischemia can be completely avoided. Additionally, the preservation of renal tissue surrounding the calyx enables the preservation of the intrasinusal segmental arteries flow, thereby avoiding their accidental closure by hemostatic sutures. Conclusions: In conclusion, Laparoscopic Nephron-Sparing Calycectomy is a safe and effective surgical procedure for the treatment of Fraley's syndrome. Consistent laparoscopic experience is required before embarking on this kind of surgery.

\section{Introduction}

In 1966, Fraley described a rare anatomic variant of the renal vascular anatomy that compresses the upper pole infundibulum resulting in an upper calyceal obstruction and dilatation, with symptoms of flank pain and hematuria [1].

The Fraley's syndrome diagnosis is confirmed by urography through which a well-defined filling defect in the infundibulum, caused by the crossing vessel compression
\end{abstract}


is detected. The upper calyx looks distended.

Various treatment methods have been employed successfully

to eliminate symptoms, usually requiring surgical

exploration. Different open nephron-sparing approaches

were described by several authors [2-4]. To

date, descriptions of nephron-sparing laparoscopic or

robot-assisted correction techniques of Fraley's syndrome

are anecdotal [5].

\section{Clinical Case}

We present the case of a 46 -year-old female with recurrent urinary tract infections with left pyelonephritis. Screening urinalysis revealed the presence of microscopic hematuria. Ultrasonography showed left upper calyx enlargement, and CT scan with urography revealed the presence of a crossing vessel at the left upper pole infundibulum resulting in a hydrocalycosis. The nuclear renal scan demonstrated that the tracer persistence collected in the left upper pole calyx even if the remnant kidney drained it promptly. Prior to surgery, a 3D reconstruction based on CT scan was performed in order to identify the location of the crossing vessel. Finally, retrograde pyelography performed in the operative room demonstrated upper pole calyx enlargement with a filling defect corresponding to a vascular impression on the infundibulum.

\section{Surgical Procedure}

A retroperitoneal pure laparoscopic approach was chosen. After placing the patient in the full-flank position, a 12-mm incision was made $1 \mathrm{~cm}$ above the iliac crest at the level of the Petit triangle. Abdominal wall and transversalis fascia were incised with scissors. Once the retroperitoneum was achieved, the space was dissected with the finger and expanded under direct vision by using a dilatation balloon. Two ports were inserted under digital guidance at the level of the anterior $(10 \mathrm{~mm})$ and posterior $(5 \mathrm{~mm})$ axillary lines. The third port $(5 \mathrm{~mm})$ was placed at the level of the tip of the 12th rib. Finally, a 12-mm Hasson port was placed at the level of the first incision so that ports were arranged in a diamond shape [6]. Retropneumoperitoneum was induced by carbon dioxide pressure fixed at $12 \mathrm{~mm} \mathrm{Hg}$. The posterior para- and peri-renal fat was dissected to gain the kidney. The upper pole was identified. It appeared redundant due to the upper calyx enlargement. Renal sinus and vascular pedicle were identified and dissected. The renal pelvis appeared normal in size, but the superior calyx was enlarged due to an obstruction generated 
by a crossing segmental vessel at the level of the calyceal

infundibulum (Fig. 1).

The crossing vessel and the upper calyceal infundibulum were than dissected.

The calyceal infundibulum was secured by reabsorbable clips

(Fig. 2) in order to avoid the risk of migration after the surgery. It was then sectioned, while the crossing vessel was preserved (Fig. 3). A smooth dissection of the calyx was performed, without any clamping of segmental vascular branches, preserving the thin coating of renal parenchyma of the upper pole of the kidney.

The calyx was completely removed in order to avoid the risk of urinary fistulas. At the end of the extirpative phase, the upper pole sinusal cavity was examined in order to identify parenchymal structures like renal papillas, Bertin Columns, and interlobar vessels

(Fig. 4). The interlobar vessels at this level were selectively coagulated avoiding intrasinusal hematoma.

To complete the procedure, the cavity was wadded with the perirenal fat and fixed with PDS 2/0 suture in order to prevent the risk of bleeding and formation of urinary fistulas.

\section{Results}

Neither intra- nor postoperative complications were recorded. The patient was discharged 3 days after surgery. The CT scan performed one month postoperatively showed no enlargement of the urinary collecting system.

\section{Discussion}

Most of anatomic and radiographic studies of the renal arterial vasculature have devoted little attention to the relation of the renal vessels to the infundibula [7-9]. Graves [10] showed that the segmental artery directed to the upper pole of the kidney - a branch of the pre-pyelic division of the main renal artery - can overlie and compress the superior calyceal infundibulum. Tests and operative finding demonstrated that the vessels responsible for the filling defects were also causing significant obstruction to urine flow from the superior infundibulum to the renal pelvis. Indeed the symptoms were undoubtedly due to a periodic over-distension of the superior calyx by the urine. The stasis of urine in the calyx predisposes to infections and segmental pyelonephritis. The treatment of choice of such an anatomical defect 
is represented by the upper pole partial nephrectomy. Notwithstanding the preservation of a huge portion of the healthy kidney, a relative loss of renal function is expected due to reversible ischemic injury that is caused when a surgery for the clamping of the arterial vasculature is performed; when a healthy portion of the kidney is removed together with the calyx; and when permanent ischemic damage of the parenchyma is involved in the resection bed suture.

In order to completely avoid the postoperative loss of renal function, new nephron-sparing techniques have been proposed. These techniques are based on the anatomical principle of the segmental distribution of the renal lobes (medullary pyramids and their own calyx). According to this concept, it is possible to describe a "pure" calycectomy, characterized by hilar access, renal sinus exploration, and isolation of a specific anatomical segment/ lobe. The interlobar vessels located between different lobes should be considered independent from calyceal structures during the surgery. Indeed, if calycectomy is performed without the clamping of renal branches, the parenchymal ischemia is completely avoided. Moreover, the preservation of renal tissue surrounding the calyx leads to the preservation of the intrasinusal segmental arteries flow, thereby avoiding their accidental closure by hemostatic sutures.

\section{Conclusion}

In conclusion, Laparoscopic Nephron-Sparing Calycectomy is a safe and effective surgical procedure for the treatment of Fraley's syndrome. If calycectomy is performed without the clamping of renal branches, parenchymal ischemia can be completely avoided. The preservation of renal tissue surrounding the calyx allows the preservation of the intrasinusal segmental arteries flow, thereby avoiding their accidental closure by hemostatic sutures.

\section{Ethics Statement}


The participant of the study gave her informed consent to publish details of the case.

\section{Disclosure Statement}

None of the authors has anything to disclose.

\section{References}

1 Fraley EE. Vascular obstruction of superior infundibulum causing nephralgia. A new syndrome.

N Engl J Med 1966; 275: 1403-1409.

2 Rassweiler J, Goezen AS: Laparoscopic partial nephrectomy in the era of robotic surgery: there is a role! Minerva Urol Nefrol 2017, Epub ahead of print.

3 D’Amico A, Lusuardi L, Ficarra V, et al: Experience in the surgical treatment of Fraley's syndrome. Eur Urol 2000; 38: 410-414.

4 Fazeli-Matin S, Streem SB: Nephron sparing management of Fraley's syndrome with a pyelopyelostomy.

J Urol 1998; 159: 487-489.

5 Armstrong JM, Soni SD, Link RE: Laparoscopic nephron-sparing treatment of upper pole infundibular obstruction due to

Fraley's syndrome. Urol Case Rep 2015; 4:

41-44.

6 Porpiglia F, Bertolo R, Amparore D, Cattaneo

G, Fiori C: Mini-retroperitoneoscopic clampless partial nephrectomy for "low-complexity" renal tumours (PADUA score $\leq 8$ ). Eur Urol 2014; 66: 778-783.

7 Baum S, Gillenwater JY: Renal artery impressions on the renal pelvis. J Urol 1966; 95: 139

145.

8 Uehara S, Yoshida S, Tanaka H, et al: Prediction of intraoperative urinary collecting system entry in patients with peripheral renal tumors undergoing partial nephrectomy: usefulness of tumor-centered multiplanar reconstruction. Urol Int 2017, Epub ahead of print.

9 Klatte T, Ficarra V, Gratzke C, et al: A literature review of renal surgical anatomy and surgical strategies for partial nephrectomy. Eur Urol 2015; 68: 980-992.

10 Graves FT: The anatomy of the intrarenal arteries and its application to segmental resection of the kidney. Br J Surg 1954; 42: 132-139. 